\title{
Vascularization of Cytochrome Oxidase-Rich Blobs in the Primary Visual Cortex of Squirrel and Macaque Monkeys
}

\author{
Anna Lena Keller, ${ }^{1}$ Almut Schüz, ${ }^{1}$ Nikos K. Logothetis, ${ }^{1,2}$ and Bruno Weber ${ }^{3}$ \\ ${ }^{1}$ Max Planck Institute for Biological Cybernetics, 72076 Tübingen, Germany, ${ }^{2}$ Imaging Science and Biomedical Engineering, University of Manchester, \\ Manchester M13 9PL, UK, and ${ }^{3}$ Institute for Pharmacology and Toxicology, University of Zurich, 8091 Zurich, Switzerland
}

The close correlation between energy supply by blood vessels and energy consumption by cellular processes in the brain is the basis of blood flow-related functional imaging techniques. Regional differences in vascular density can be detected using high-resolution functional magnetic resonance imaging. Therefore, inhomogeneities in vascularization might help to identify anatomically distinct areas noninvasively in vivo. It was reported previously that cytochrome oxidase-rich blobs in the striate cortex of squirrel monkeys are characterized by a notably higher vascular density ( $42 \%$ higher than interblob regions). However, blobs have so far never been identified in vivo on the basis of their vascular density. Here, we analyzed blobs of the primary visual cortex of squirrel monkeys and macaques with respect to the relationship between vascularization and cytochrome oxidase activity. By double staining with cytochrome oxidase enzyme histochemistry to define the blobs and collagen type IV immunohistochemistry to quantify the blood vessels, a close correlation between oxidative metabolism and vascularization was confirmed and quantified in detail. The vascular length density in cytochrome oxidase blobs was on average $4.5 \%$ higher than in the interblob regions, a difference almost one order of magnitude smaller than previously reported. Thus, the vascular density that is closely associated with local average metabolic activity is a structural equivalent of cerebral metabolism and blood flow. However, the quantitative differences in vascularization between blob and interblob regions are small and below the detectability threshold of the noninvasive hemodynamic imaging methods of today.

\section{Introduction}

Cytochrome oxidase (CO) blobs in the primary visual cortex V1 of macaques were described $>25$ years ago (Horton and Hubel, 1981). Because their functions remain elusive (Sincich and Horton, 2005), noninvasive functional imaging techniques could be a valuable tool for research in this field. Blobs are characterized anatomically as zones of increased $\mathrm{CO}$ activity in all cortical layers except IVa and IVc of area V1 (Hendrickson et al., 1981; Horton and Hubel, 1981) and most prominent in lower layer II/III. Blobs have been described for some mammalian species, such as the cat (Murphy et al., 1995), and all primates examined so far [e.g., squirrel monkeys (Fitzpatrick et al., 1983; Carroll and WongRiley, 1984), macaques (Horton and Hubel, 1981), and humans (Horton and Hedley-Whyte, 1984; Adams et al., 2007)]. Although there is converging evidence that they are involved in the processing of color, the exact function of blobs remains obscure. Two recent publications (Sincich and Horton, 2005; Lu and Roe, 2008) have provided comprehensive overviews of efforts undertaken so far to clarify this issue. According to these investigators,

\footnotetext{
Received June 1, 2010; revised Nov. 5, 2010; accepted Nov. 7, 2010.

This work was supported by the Max Planck Society and by Swiss National Science Foundation Grant PP0033110751. We thank Prof. Dr. Ernst Pöppel and the University of Munich for providing us with the monkeys used in this study. We thank Kevan Martin and Johannes Reichold for their valuable comments. We thank Monica Pawelec for English corrections and editing.

This article is freely available online through the J Neurosci Open Choice option.

Correspondence should be addressed to Anna Lena Keller, Max Planck Institute for Biological Cybernetics, Spemannstrasse 41, 72076 Tübingen, Germany. E-mail: anna.keller@tuebingen.mpg.de.

DOI:10.1523/JNEUROSCI.2765-10.2011

Copyright $\odot 2011$ the authors $\quad 0270-6474 / 11 / 311246-08 \$ 15.00 / 0$
}

the conflicting results achieved with methods such as electrophysiology (Livingstone and Hubel, 1988; Ts'O and Gilbert, 1988; Yoshioka and Dow, 1996) and optical imaging (Landisman and Ts'O, 2002) are not necessarily exclusive.

One undisputed fact is the increased oxidative metabolism of the blobs, as shown by CO histochemistry. Such metabolic activity patterns have been linked to certain cortical pathways in primates (Fitzpatrick et al., 1983; Levitt et al., 1995) and correlated with capillary density in rodents (Borowsky and Collins, 1989; Woolsey and Rovainen, 1991). The relationship between vascularization and $\mathrm{CO}$ activity has been confirmed by subsequent studies in primates (Zheng et al., 1991; Weber et al., 2008). Specifically, Zheng and colleagues found a difference of $42 \%$ in vascular density between blobs and interblobs and also more of the large perpendicular vessels within the blobs in Saimiri. Our own recent study found a close correlation between vascularization and laminar organization of CO activity in $\mathrm{V} 1$ of macaques (Weber et al., 2008). Layer IVc, with the highest vascular density, was different from the other cortical layers by $\sim 30 \%$, rendering it clearly discernable in high-resolution spin-echo blood oxygenation level-dependent functional magnetic resonance imaging (SE BOLD fMRI) (Goense et al., 2007). Consequently, given the allegedly even higher blob/interblob difference found by Zheng and colleagues and a blob size of $\sim 0.25 \mathrm{~mm}$, blobs should be detectable using high-resolution MRI. However, to our knowledge, blobs have never been identified using MRI technology. This led to the present study, in which a careful reappraisal of CO activity, vascular density, and evaluation of perpendicular vessels was performed in V1 of macaque and squirrel monkey. We did 
indeed find a difference in vascular density between blob and interblob regions, but one order of magnitude smaller (Saimiri, 4.6\%; Macaca, 4.4\%) than published previously. This smaller difference is in accordance with the vascularization and $\mathrm{CO}$ activity differences found across cortical layers, which span a considerably larger range.

\section{Materials and Methods}

Animals. The brains of two adult squirrel monkeys (Saimiri sciureus; one male, 5 years of age; one female, 15 years of age) and two adult macaques (Macaca nemestrina; two males, 13 and 15 years of age) were used in this study. The colonies of these animals were bred at the Ludwig Maximilian University of Munich, where they were involved in long-term observations concerning their circadian rhythms and social behavior. No invasive experiments were performed. Animals were killed with pentobarbital $(120 \mathrm{mg} / \mathrm{kg}$; in accordance with the guidelines of the American Veterinary Association Panel on Euthanasia and with the recommendations of the National Institutes of Health Guide for the Care and Use of Laboratory Animals). All procedures were approved by the local authorities (Regierungspräsidium) and are also in compliance with the guidelines of the European community (EUVD 86/609/EEC) for the care and use of laboratory animals.

Histochemistry. The macaques were perfused transcardially under deep sodium pentobarbital anesthesia with $8 \mathrm{~L}$ of $0.9 \%$ body temperature heparinized $0.1 \mathrm{M}$ PBS, followed by $2 \mathrm{~L}$ of cold $2 \%$ paraformaldehyde (PFA) in $0.1 \mathrm{M}$ phosphate buffer (PB), then $4 \mathrm{~L}$ of $4 \%$ PFA (Roti Histofix; Carl Roth) in $0.1 \mathrm{M} \mathrm{PB}$, and finally $1 \mathrm{~L}$ of $10 \%$ sucrose (Sigma-Aldrich) in $0.1 \mathrm{M}$ PB. The procedure and the chemicals were the same for the squirrel monkeys, but with only $2 \mathrm{~L}$ of $0.9 \%$ body temperature heparinized $0.1 \mathrm{M}$ PBS, followed by $0.5 \mathrm{~L}$ of cold $2 \%$ PFA in $0.1 \mathrm{M} \mathrm{PB}$, then $1 \mathrm{~L}$ of $4 \%$ PFA in $0.1 \mathrm{M} \mathrm{PB}$, and finally $0.25 \mathrm{~L}$ of $10 \%$ sucrose in $0.1 \mathrm{M} \mathrm{PB}$. The brain was removed from the skull immediately after perfusion. The primary visual cortex of one hemisphere was removed, slightly flattened with a strip of gauze pressing the piece of tissue with the cortical surface against a glass slide, and postfixed overnight in 4\% PFA containing $10 \%$ sucrose. The flattening procedure resulted in tangential sections from the lateral surface of the primary visual cortex, approximately parallel to the cortical layers. The other hemisphere was not flattened and was sectioned horizontally. Sections in which the cutting plane was vertical to the cortical surface of $\mathrm{V} 1$ were taken for layerwise investigations of $\mathrm{CO}$ intensity. The following steps were kept as short as possible to preserve the best obtainable $\mathrm{CO}$ activity. For cryoprotection before sectioning, the brain blocks were placed in a series of sucrose solutions of increasing concentration $(10,20$, and $30 \%)$ in 0.1 M PB until they sank. Sixty-micrometer-thick (previously calibrated) tangential and horizontal sections were then cut on a freezing sliding microtome (Microm HM 440E), for the tangential sections, from the cortical surface to the white matter. All sections were processed first for CO staining (Wong-Riley, 1979, 1989; Wong-Riley and Welt, 1980; Carroll and Wong-Riley, 1984). Incubation times varied between 4 and $8 \mathrm{~h}$, depending on the freshness of the tissue, the time required for cryoprotection, respectively. The sections optimally showing the blobs were afterward processed for fluorescence immunohistochemistry to label collagen type IV to visualize the vasculature (Hamann et al., 1995; Fukuda et al., 2004; Weber et al., 2008). They were collected in $0.1 \mathrm{M} \mathrm{PB}$ and then transferred to staining solution consisting of $0.05 \%$ $\operatorname{DAB}\left(3,3^{\prime}\right.$-diaminobenzidine tetrahydrochloride), $0.04 \%$ cytochrome $c$ from horse heart, and 3\% sucrose (all from Sigma-Aldrich) in $0.1 \mathrm{M} \mathrm{PB}$. Incubation was performed free-floating with gentle agitation at $37^{\circ} \mathrm{C}$ under visual control until the contrast between blobs and interblobs was found to be sufficient. The staining reaction was stopped by washing three times for $5 \mathrm{~min}$ each time in $0.1 \mathrm{M}$ PB. The sections were blocked to prevent nonspecific binding of the antibody by $1 \mathrm{~h}$ incubation at room temperature in $10 \%$ normal goat serum (collected and provided by the Max Planck Institute for Developmental Biology, Tübingen, Germany), 2\% BSA (Sigma-Aldrich), and 0.4\% Triton X-100 (Carl Roth). The primary antibody (monoclonal anti-collagen type IV; clone col-94; SigmaAldrich) was added to fresh blocking solution (same preparation as above) at a dilution of 1:500 and the sections were incubated for $72 \mathrm{~h}$ at $4^{\circ} \mathrm{C}$ (Zaitsev et al., 2005). They were then washed three times, $5 \mathrm{~min}$, in
$0.1 \mathrm{M}$ PBS before being incubated another $72 \mathrm{~h}$ at $4^{\circ} \mathrm{C}$ in the dark in $0.1 \mathrm{M}$ PBS containing a 1:500 dilution of the secondary antibody (Cy-3conjugated goat anti-mouse IgG $(\mathrm{H}+\mathrm{L})$; Jackson ImmunoResearch). After a last series of three washes in $0.1 \mathrm{M}$ PBS, the stained sections were mounted on glass slides and coverslipped wet in polyvinyl alcohol (Mowiol 4-88; Hoechst) containing 4\% DABCO (1,4-diazobicyclooctane) (Merck) as an antifading reagent to preserve fluorescence.

Microscopy. Cy-3 and bright-field images of identical fields of view were acquired using a fluorescence microscope (Axiophot; $5 \times$ objective; Carl Zeiss) equipped with a monochrome CCD camera (Axiocam MRm, controlled by Axiovision 4.3; Zeiss).

Nomenclature. We followed the naming scheme for layers of the primary visual cortex of Brodmann (1904-1905), with the additional subdivision of layer IVc into two sublayers (namely layer IVc-a and IVc-b) as introduced by Polyak (1957).

Data analysis. Most measurements were made on micrographs taken from the one or two tangential sections through layer II/III that showed the blobs most clearly (see Fig. $1 A$ ). Twenty two-channel images (see Fig. 2), $\sim 1.1 \times 1.4 \mathrm{~mm}$ in size, were analyzed per animal. The original Cy3labeled blood vessel micrographs were filtered, thresholded, and inverted to yield binary images of the vasculature that were appropriate for additional processing (Matlab; The MathWorks) to yield single-pixel-wide midline traces of the vessels (see Fig. $3 A-C$ ). Larger perpendicular blood vessels were filled manually on the inverted images (see Fig. $3 B, B^{\prime}$ ) to avoid measuring their circumference instead of their length. The total projected vessel length was measured on these eroded traces; the length density (in millimeters per cubic millimeter) was then stereologically corrected (taking into account the bias induced by the finite section thickness) (Russ and Dehoff, 2000). Details of the density calculation are given in the appendix of Weber et al. (2008). Blobs were identified by visual inspection and region of interest (ROI) borders were manually drawn where staining intensity dropped to background. To test the influence of the manual ROI definition, the ROIs were automatically eroded and dilated in steps of $10 \%$ of the original ROI area. For the interblob regions, the total vessel length and volume were determined in the whole image and the sums of the lengths and volumes of the blobs from that respective image were subtracted.

To ensure that the half-automated image processing yielded the same results as a manual delineation of the vasculature (Zheng et al., 1991), two representative images per animal were drawn in Adobe Photoshop CS with a pen tablet (Bamboo Fun; Wacom) on the basis of the anti-collagen staining, further processed as described previously, and the results compared (see Fig. 3D). Only minor deviations of these differently generated vessel courses from each other could be observed, and after thorough comparison this effect was rated as negligible. Zheng and colleagues have chosen to define vascular density in millimeters per square millimeter rather than in millimeters per cubic millimeter. It is important to note that the relative difference in percentage between the investigated subregions does not depend on the chosen unit.

For the layerwise evaluation of the CO staining intensity, micrographs from the horizontal sections (like Fig. $1 \mathrm{~A}$, but monochrome) were taken, and ROIs for the cortical layers and for the blob and interblob regions in lower layer III were defined. For the tangential evaluation, the same CO-stained bright-field micrographs and blob ROIs as described for the blood vessel quantification were taken. The layerwise gray values from the horizontal sections were measured and normalized to make them consistent with the $\mathrm{CO}$ activity measurements from previous work (Weber et al., 2008). More precisely, the mean gray value across all layers was normalized to be the same in both studies. The gray values measured on tangential sections that originate only from blob and interblob regions were then normalized to be comparable with the data measured on horizontal sections (mean of blob and interblob from each respective micrograph measured tangentially matched the mean of blob and interblob value measured horizontally).

The evaluation of perpendicular blood vessels was performed on the original Cy3-labeled fluorescent images. They were not defined by their diameter, because some feeding arteries and draining veins already ramify to capillaries in the upper cortical layers (Duvernoy et al., 1981) and could therefore be rather thin when reaching lower layer III, where the 
measurements were made. Evaluated vessels ran perpendicularly to the cutting plane and therefore appeared as open (larger vessels) or filled (smaller vessels) circles and were surrounded by the characteristic empty space known as "Zirkumvasaler Kapillarfreier Raum" (Pfeifer, 1930) or "Pfeifer space" as cited by several other authors (Lierse, 1963; Saunders and Bell, 1971; Duvernoy et al., 1981) (see Fig. $2 \mathrm{~B}$ ). A boundary box was manually drawn around each perpendicular vessel on the anti-collagen-stained vessel images. Within this boundary box, an ellipse was automatically fitted to the vessel (least-square fit). The minor axis of the ellipse was taken as the estimate of the vessel diameter. An overlay with the blob ROIs provided the definition of blob and interblob vessels. These analyses were performed with Matlab (The MathWorks).

\section{Results}

\section{Number and distribution of blobs}

In both primate species, individual blobs appeared to be mostly round or elliptical in shape and were uniformly distributed in tangential sections through layer III of the primary visual cortex V1 (Figs. 1 A, 2A). On average, a single blob occupied an area of $2.6 \times 10^{4} \pm 0.06 \times$ $10^{4} \mu \mathrm{m}^{2}$ in Saimiri and $3.8 \times 10^{4} \pm 0.1 \times 10^{4} \mu \mathrm{m}^{2}$ in Macaca and occurred with a frequency (see Fig. $4 A$, left-hand side) of $6.8 \pm 0.26 / \mathrm{mm}^{2}$ ( \pm SEM; $n=406$ blobs in $N=40$ micrographs $)$ and $7.4 \pm 0.27 / \mathrm{mm}^{2}( \pm$ SEM; $n=443, N=40)$. Our data revealed no statistically significant difference between the species with respect to the spatial density of blobs, whereas the difference with respect to the mean size of the blobs was significant (independent $t$ test, $t=9.37, p<0.001)$.

\section{Vascular density}

The larger blood vessels feeding and draining the capillary bed (i.e., arteries and veins) are running perpendicular to the cortical surface. The number and the mean diameter of these vertical blood vessels was different between squirrel and macaque monkeys (independent $t$ test, $t=-5.42, p<0.001$ ). We counted $28.2 \pm 0.64$ vessels $/ \mathrm{mm}^{2}$ in squirrel monkeys ( \pm SEM; $n=1675$ vessels in $N=40$ micrographs) and $23.6 \pm 0.57$ vessels $/ \mathrm{mm}^{2}$ in macaques ( \pm SEM; $n=1407 ; N=40$ ) (see Fig. $4 A$, middle). The mean diameter of these vessels was $23.8 \pm 0.16 \mu \mathrm{m}$ in squirrel monkeys and $26.1 \pm 0.19 \mu \mathrm{m}$ in macaques (independent $t$ test, $t=-8.98, p<0.0001$ ) (see Fig. 4A, right). Large perpendicular cortical vessels were preferentially positioned in the interblob regions in both species (see Fig. $4 C$, left-hand side), with $24.9 \pm 1.1$ vessels $/ \mathrm{mm}^{2}$ in blobs and $28.9 \pm 0.7$ vessels $/ \mathrm{mm}^{2}$ in interblobs in Saimiri and $21.0 \pm 1.1$ vessels $/ \mathrm{mm}^{2}$ in blobs and $24.7 \pm 0.6$ vessels $/ \mathrm{mm}^{2}$ in interblobs in Macaca ( \pm SEM; $N=40$; paired $t$ tests, $\left.t_{\text {Saimiri }}=-3.6, t_{\text {Macaca }}=-3.0, p<0.01\right)$. This corresponded to a ratio of blob to interblob vessels of 1:1.2 in both species if all vertical vessels, of which the smallest had a diameter of $8 \mu \mathrm{m}$, were taken into account. However, increasing this threshold changed the ratio (see Fig. $4 E, F$ ). Equal numbers of vertical blood vessels were measured in both areas when only those with a diameter $>25 \mu \mathrm{m}$ (Saimiri) or $>28 \mu \mathrm{m}$ (Macaca) were counted. By raising the threshold further, the ratio was reversed (slightly higher number of perpendicular vessels within blobs).

Next, we analyzed whether the overall vascular density followed the distribution of the perpendicular vessels. We found that less perpendicular vessels in the blobs correlated with a

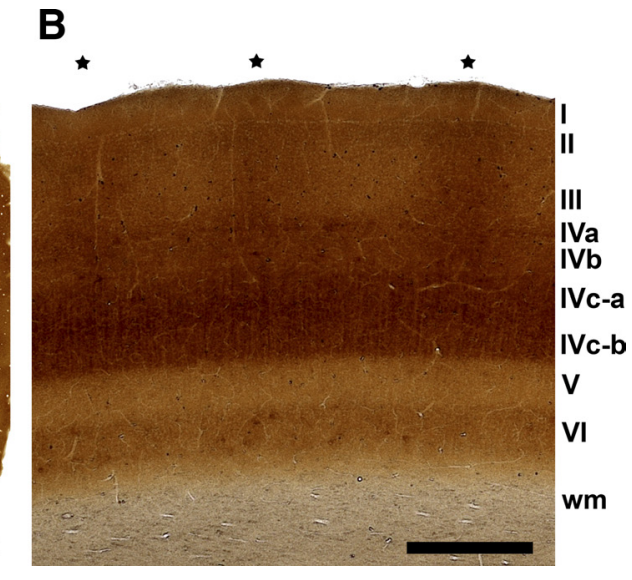

Figure 1. Micrographs of $\mathrm{CO}$ staining in tangential and horizontal sections of primate $\mathrm{V} 1$; both sections are $60 \mu \mathrm{m}$ thick and cut frozen on a sliding microtome. $A$, Low-power magnification overview of a $C 0$-stained tangential squirrel monkey section through of the primary visual cortex, showing the regular distribution and mostly round appearance of the blobs. Scale bar, 1000 $\mu \mathrm{m} . \boldsymbol{B}$, Micrograph of a $\mathbf{C O}$-stained horizontal macaque monkey section through the primary visual cortex (asterisks denote the positions of the blobs visible in layer III; roman numerals indicate the cortical layers). Scale bar, $500 \mu \mathrm{m}$.

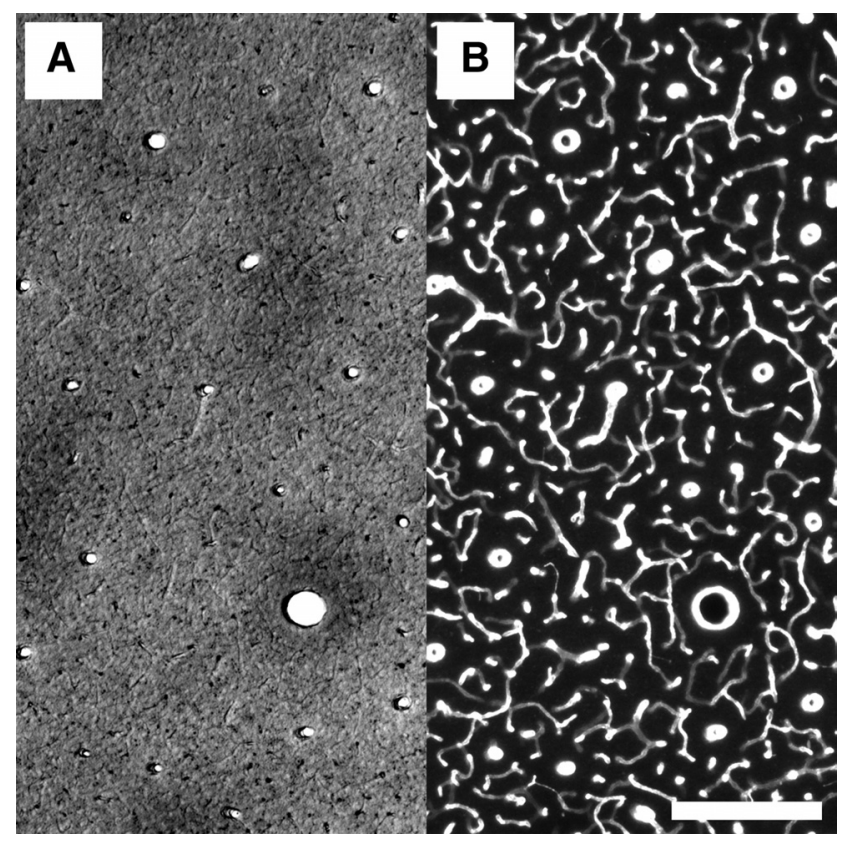

Figure 2. Quality of individual staining for $\mathrm{CO}$ activity and blood vessels in double-stained specimen, here a 60 - $\mu \mathrm{m}$-thick tangential section from a squirrel monkey. $A, A 50 \times$ magnified bright-field micrograph from a similar section as in Figure 1 showing some $\mathrm{CO}$ blobs. $\boldsymbol{B}$, Fluorescence micrograph from the same area of the same section as in Figure $3 A$, showing the blood vessels stained with a primary antibody against collagen type IV and a secondary antibody labeled with Cy3. Scale bar, $200 \mu \mathrm{m}$.

higher vascular length density. Figure 2 shows an example of a double-stained sample. CO staining was used to define the blobs (Fig. 2A) and fluorescent anti-collagen staining to label and quantify the blood vessels (Figs. 2B, 3). This analysis showed a generally higher vascular density in the Saimiri for all subareas examined (Fig. 4B) [i.e., blobs (Saimiri, $619.7 \pm 12.0 \mathrm{~mm} / \mathrm{mm}^{3}$; Macaca, $545.0 \pm 8.3 \mathrm{~mm} / \mathrm{mm}^{3}$ ), interblobs (Saimiri, $592.7 \pm$ $10.1 \mathrm{~mm} / \mathrm{mm}^{3} ;$ Macaca, $522.1 \pm 9.1 \mathrm{~mm} / \mathrm{mm}^{3}$ ), and overall (Saimiri, $597.1 \pm 10.3 \mathrm{~mm} / \mathrm{mm}^{3}$; Macaca, $528.4 \pm 8.8 \mathrm{~mm} /$ $\mathrm{mm}^{3}$; $\pm \mathrm{SEM} ; N=40$; independent $t$ tests, $\left.\left.t_{\text {all }} \sim-5, p<0.001\right)\right]$. Moreover, the vascular length density in the blobs was consistently higher than in the surrounding interblob regions (Fig. 4D). 

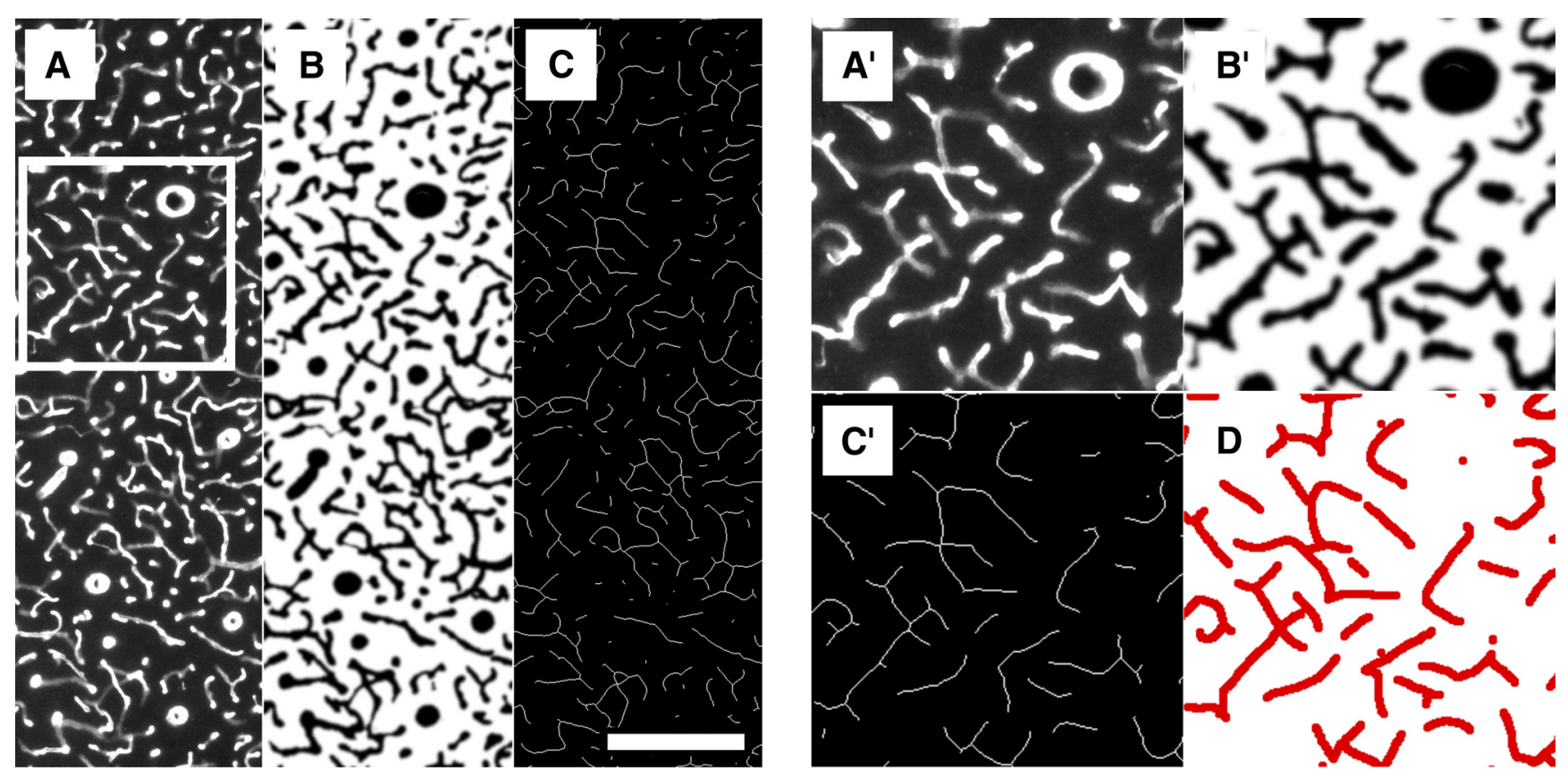

Figure 3. Example of the image-processing protocol of the fluorescent blood vessel images from the same sample as in Figure 2 . The anti-collagen immunohistochemistry produced images of high quality that allowed for the semiautomated approach in this study. $\boldsymbol{A}$, Raw image of an anti-collagen fluorescently stained section. $\boldsymbol{B}$, The same image as in $\boldsymbol{A}$ after visually guided semiautomated filtering, thresholding and binarization. $\boldsymbol{C}$, The same image as in $\boldsymbol{B}$ after erosion of the vasculature to a line set where the blood vessels are represented by lines of a single pixel width. Scale bar, $200 \mu \mathrm{m} . \boldsymbol{A}^{\prime}, \boldsymbol{B}^{\prime}, \boldsymbol{C}^{\prime}$, Enlarged subareas indicated in $\boldsymbol{A}$ in greater detail in the different image modalities described previously. $\boldsymbol{D}$, Manual delineation of the raw fluorescent micrograph shows almost no deviation from the course of the eroded lines in $\boldsymbol{C}^{\prime}$.

On average, the length density of all blood vessels within blobs was $4.6 \%$ higher than in interblob regions in squirrel monkeys and $4.4 \%$ higher in blobs compared with interblobs in the macaque $\left(N=40\right.$; paired $t$ tests, $t_{\text {Saimiri }}=6.1$ and $t_{\text {Macaca }}=8.3, p<$ $0.0001)$. It has to be noted that the measured length density should be unbiased by the unequal distribution of large perpendicular vessels. Whether the measurement of the volume fraction would yield another relationship of blob to interblob vascularization remains an open question. Analysis of the influence of manually identified blob boundaries on the difference in vascular density between blobs and interblobs revealed the existence of a narrow rim surrounding the blobs with an even higher vascular density than within the blobs (Saimiri, $624.5 \pm 12.4 \mathrm{~mm} / \mathrm{mm}^{3}$; Macaca, $554.7 \pm 9.6 \mathrm{~mm} / \mathrm{mm}^{3}$ ). However, this region seems not to belong to the blobs in the strict sense, as no strong CO staining was apparent. When only this rim was compared with the respective interblob area (Saimiri, $587.3 \pm 10.2 \mathrm{~mm} / \mathrm{mm}^{3}$; Macaca, $516.3 \pm 9.4 \mathrm{~mm} / \mathrm{mm}^{3}$ ), the difference was found to be $6.3 \%$ for squirrel monkey and $7.4 \%$ for macaques $(N=40$; paired $t$ tests, $t_{\text {Saimiri }}=5.0$ and $\left.t_{\text {Macaca }}=7.9, p<0.0001\right)$. Nevertheless, there were no significant differences between blobs and blob surroundings or between the original and the smaller interblob region.

\section{Cytochrome oxidase activity}

The fact that the cytochrome-rich blobs showed a higher microvascular density compared with interblob regions was consistent with the fact that $\mathrm{CO}$ activity is a marker for the degree of oxidative metabolism. However, this difference in vascular density was relatively small, particularly when compared with the differences between the cortical layers (Weber et al., 2008). To better understand this discrepancy, we quantified the $\mathrm{CO}$ activity in more detail.

The difference in $\mathrm{CO}$ staining intensity of blobs compared with interblobs in the horizontal sections was slightly more pronounced in the macaque [Fig. 5A, left-hand side: Saimiri: $0.05 \pm$
0.006 arbitrary units (a.u.), $N=10$; Macaca: $0.08 \pm 0.010$ a.u., $N=12$; \pm SEM; independent $t$ test, $t=2.53, p<0.05]$. Visual inspection indicated that the difference between blobs and interblob regions was smaller compared with the differences across cortical layers (Fig. 1 $A, B$ ). In relative terms, the blob-interblob difference in CO activity was $8 \%$ in Saimiri and 10\% for Macaca. This was considerably smaller than the CO activity range across cortical layers, with a respective maximum difference of $64 \%$ in Saimiri between layer I and the upper subdivision of layer IVc (i.e., layer IVc-a) and 54\% in Macaca between layer V and layer IVc-a.

Measurements on the tangential sections yielded a more pronounced blob-interblob difference in CO activity in Saimiri compared with Macaca (Fig. 5A, right-hand side: Saimiri: $0.07 \pm$ 0.002 a.u., $N=40$; Macaca: $0.06 \pm 0.003$ a.u., $N=40$; \pm SEM; independent $t$ test, $t=-3.13, p<0.01$ ).

The overall correlation of $\mathrm{CO}$ activity and vascular density in blobs and interblobs was comparable with that observed for the different cortical layers (Fig. 5C), despite being measured in sections of different orientations. As a consequence, the regionally different staining intensities, whether between layers or other subareas, reflected differences in vascularization and vice versa (Fig. 5B).

\section{Discussion}

The energy cost of the brain is the sum of a variety of complex biophysical processes (Attwell and Laughlin, 2001; Jolivet et al., 2009). The local metabolic rate is reflected in regional glucose utilization (Humphrey and Hendrickson, 1983) and, partly, in regional CO activity (Borowsky and Collins, 1989; Wong-Riley, 1989). The proportional consumption of each cerebral element with respect to total brain energy requirements is not clear, and correlating cellular and synaptic density with energy metabolism and vascular density is not straightforward (Baborie and Kus- 
A

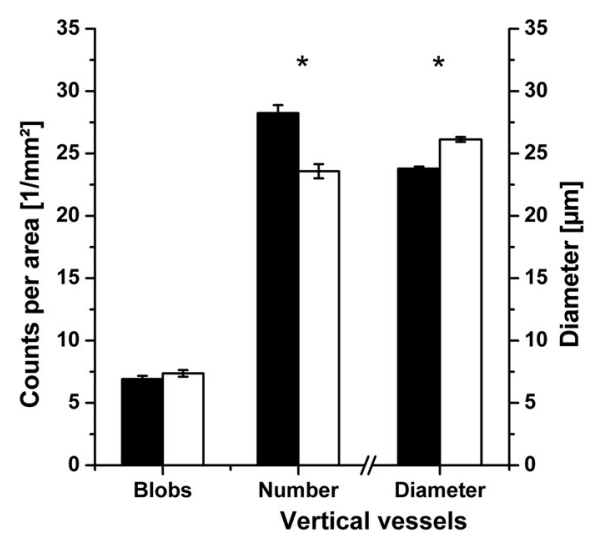

C

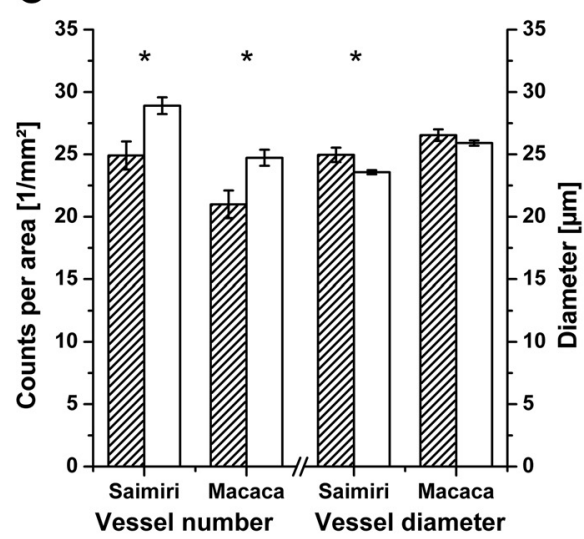

E

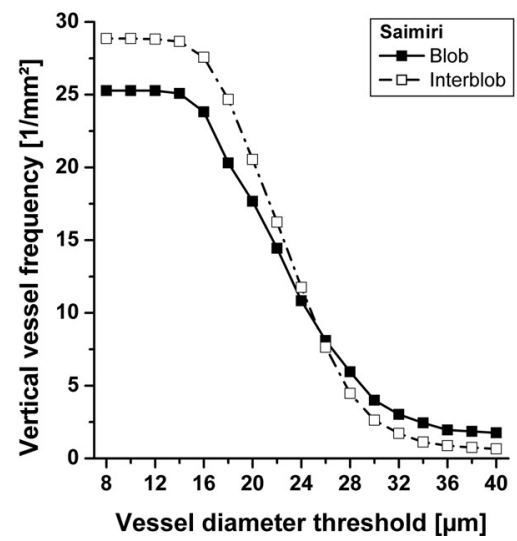

B

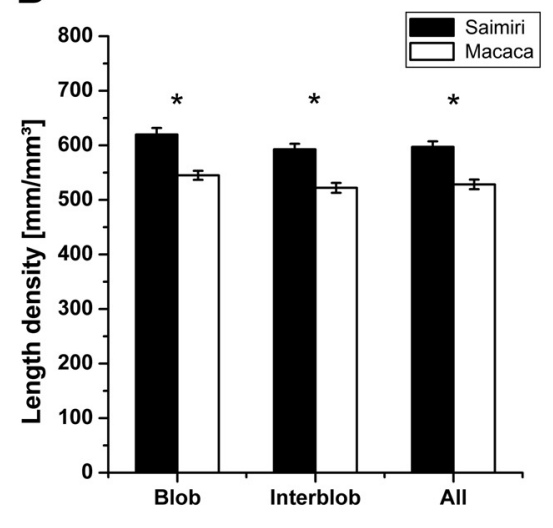

D

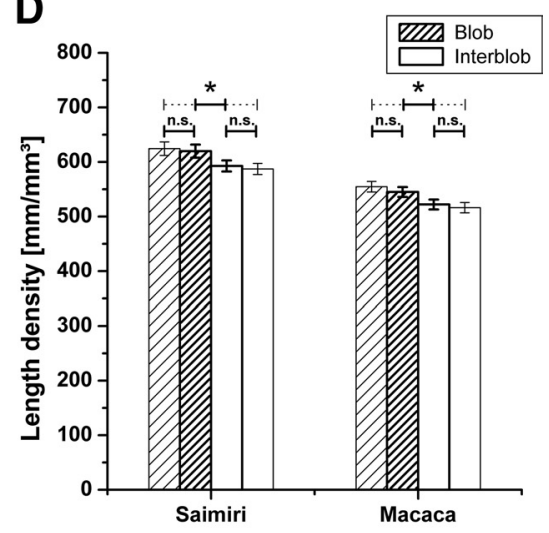

$\mathbf{F}$

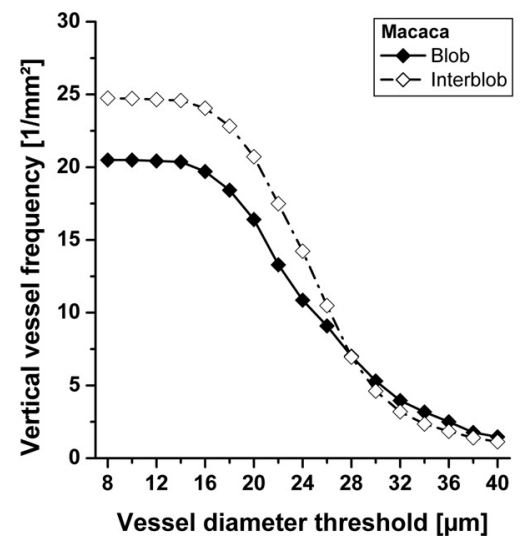

chinsky, 2006; Weber et al., 2008; Tsai et al., 2009). However, metabolic activity is mirrored by regional vascularization ( $\mathrm{Pa}-$ tel, 1983; Borowsky and Collins, 1989; Weber et al., 2008). The present study is consistent with a strong correlation between oxidative metabolism and vascularization. It is becoming increasingly clear that neuronal activity, cerebral metabolism, and blood flow constitute a tightly coupled ensemble, which is reflected structurally by a vascular density that is highly correlated with local average metabolic activity.

One consequence of this relationship is that brain regions definable anatomically by enzyme histochemistry can be identified in vivo using high-resolution hemodynamic imaging. Layer IVc of the macaque's primary visual cortex displays a vascular density that is $\sim 30 \%$ higher than in the other layers (Weber et al., 2008). This vascularization difference results in discernability of layer IVc in highresolution SE BOLD fMRI (Goense et al., 2007). Zheng et al. (1991) have estimated the difference in vascular density between blobs and interblobs in primate $\mathrm{V} 1$ to be $42 \%$. Given such a large difference in vascular density, and their relatively large size, blobs should be discernible by stateof-the-art tomographic functional imaging. However, to our knowledge, this has never been shown and the present study now provides the reason for this.

In agreement with Zheng and colleagues, we found a significant difference between blob and interblob regions, but one order of magnitude smaller. Although interindividual variations between the two animals of each species studied here were marginal, it remains possible that other individuals might show more pronounced differences. However, a previous study on layerwise vascularization (Weber et al., 2008) showed very little varia-

Figure 4. Top panel, Interspecies differences in numbers of blobs, numbers and diameters of perpendicular vessels, and density of vascularization. The asterisks indicate a statistically significant difference, and the error bars show SEM. $A$, The histogram shows counts per area (left $y$-axis) of blobs and vertical blood vessels and mean diameter (right $y$-axis) of vertical blood vessels in squirrel and macaque monkeys. The number of blobs is $8.1 \%$ higher in Macaca (difference not significant), whereas the number of perpendicular vessels is $19.5 \%$ higher in squirrel monkeys compared with macaques. The mean diameter of these vertical vessels is $9.6 \%$ greater in macaques. $\boldsymbol{B}$, The histogram shows the vascular length density in millimeters per cubic millimeter in blobs, interblobs, and overall for the two species investigated. The length density of blood vessels in tangential sections through layer III is significantly different between squirrel and macaque monkeys in all investigated subareas, and the squirrel monkey has in general a $\sim 11.5 \%$ higher vascular density than the macaque. Middle panel, Numbers of perpendicular vessels and density of vascularization between blobs and interblobs in primates' $\mathrm{V}$. The asterisks indicate a statistically significant difference, and the error bars show SEM. C, The histogram shows counts per area (left $y$-axis) and mean diameter (right $y$-axis) of vertical blood vessels in blobs and interblobs of squirrel and macaque monkeys. The left half of the histogram shows the number of perpendicular vessels, and the right-hand side shows the diameters of the vessels in blobs and interblobs. In both species investigated, there are significantly more perpendicular vessels per area in the interblob areas (i.e., 16.1\% more in Saimiriand 17.6\% more in Macaca). The diameter of these is only a little larger within the blobs, $5.9 \%$ in Saimiri and 2.4\% in Macaca. D, As shown in the histogram, the vascularization of blobs and interblobs is significantly different in both species investigated. The vascular density inside the blobs is $4.6 \%$ higher in Saimiri and $4.4 \%$ higher in Macaca compared with the interblob region. The sparsely hatched bar at far left shows

vascularization in the immediate surroundings of the blobs, measured in a rim around each blob amounting to $50 \%$ of the area of the individual blob. The thinly outlined bar at far right represents vascularization in the interblob region without the surroundings. The vascularization difference between blob surround and respective interblob region is $6.3 \%$ in Saimiri and $7.4 \%$ in Macaca. Bottom panel, Cumulative curve showing the number of vertical vessels per area in blobs and interblobs in relation to the threshold diameter applied for the quantification. Note the crossing of the traces at a certain diameter value. Below this threshold there are more perpendicular vessels in the interblob regions, and above it the ratio reverses and more vertical vessels are found within blobs. $E$, In the squirrel monkey, the threshold diameter is $25 \mu \mathrm{m}$. $\boldsymbol{F}$, In the macaque monkey, the threshold diameter is $28 \mu \mathrm{m}$. 
A

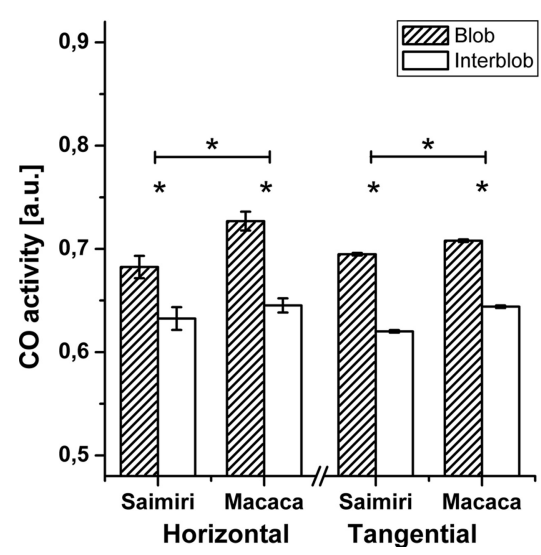

B

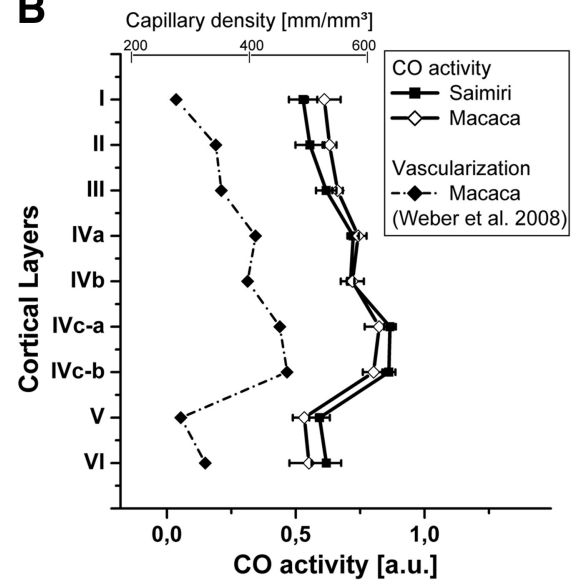

C

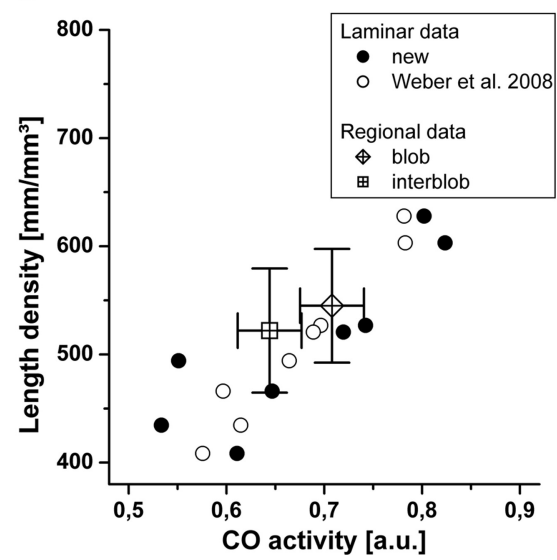

Figure 5. Relative $\mathrm{C} 0$ activity in blobs and interblobs and across the cortical layers and its correlation to vascular density. Data from Weber et al. (2008) are added. The C 0 staining intensity was measured as gray values in black-and-white images from vertical sections (as in Fig. 1B) and tangential sections (as in Fig. $2 A$ ). C 0 activity was normalized as described in Materials and Methods to allow comparison of images from different section orientations, different overall staining intensities, and therefore different image acquisition parameters. The asterisks indicate a statistically significant difference, and the error bars show SEM. $A$, The histogram shows $\mathrm{C} O$ activity in arbitrary units for blobs and interblobs in horizontal and tangential sections from squirrel and macaque monkeys. It is significantly higher in blobs than in interblobs in both species investigated, which is already clearly visible by eye in Figures $1 A$ and $2 A$. The differences are comparable across all acquisition modalities. In horizontal sections, it is $7.9 \%$ in Saimiri and $9.5 \%$ in Macaca, and in tangential sections, $12.0 \%$ in Saimiri and $9.9 \%$ in Macaca. B, The solid traces show the relative C 0 activity (bottom axis) across all cortical layers of the primary visual cortex in squirrel and macaque monkey. The course of the traces could already be assumed after the visual examination of Figure $1 B$. It also mirrors the capillary density shown in the dashed trace [top axis; only data for the macaque available from the study by Weber et al. (2008)]. C, Correlation between $\mathrm{C} O$ activity and vascular density across cortical layers, emphasizing the close relationship between $\mathrm{C} 0$ activity and vascular length density (filled circles) and the good agreement with previously published data (open circles) (Weber et al., 2008). The data from blob and interblob areas on tangential sections (open squares and diamonds; $x$ and $y$ error bars show SD) also lie well within the same range.

tion across four animals. Additionally, both CO activity and vascular density measured for blobs and interblobs lie well within the range of the values for the cortical layers (Fig. 5C). More specifically, the relative range across cortical layers is similar for CO activity [54\%, data from this study; $36 \%$ from the study by Weber et al. (2008)] and vascular density [55\%, data from the study by Weber et al. (2008)]. The difference in CO activity between blobs and interblobs is considerably smaller $(\sim 10 \%)$ and accordingly the difference in vascularization can also be expected to be smaller.

The discrepancy between these studies is most likely caused by the different methods used to visualize the blood vessels. Zheng et al. (1991) analyzed CO images to quantify the vascular density. They used transillumination with the condenser aperture stopped down to enhance vessel contrast. It is possible that this might have worked well for the darker CO stained blobs, but not for the unstained interblob region, which could result in underestimation of the vascular density in the interblobs. Our combined fluorescent and bright-field microscopic double-staining approach is unaffected by such bias. The volumetric length density values (in millimeters per cubic millimeter) can easily be converted to the areal length density used by Zheng et al. (1991) (in millimeters per square millimeter). Indeed, we find the absolute numbers for blob regions to be very similar in the two studies, whereas the interblob values of Zheng et al. (1991) are considerably smaller than ours.

Both element density (i.e., sum of neurons, synapses, and glia) and number of incoming connections of the blobs is not as large as that of layer IVc (O'Kusky and Colonnier, 1982; Carroll and Wong-Riley, 1984), although spontaneous activity of blob neurons seems to be comparable with layer IVc (Livingstone and Hubel, 1984). The latter might be related to the lack of orientation selectivity in most blob neurons, which would make them more active in most natural viewing conditions (Livingstone and Hubel, 1984). This could in turn cause the on-average stronger $\mathrm{CO}$ staining reactivity of mitochondria in dendrites and axon terminals within blobs, whereas synaptic (Carroll and WongRiley, 1984) and cellular densities (Trusk et al., 1990) are not different between blobs and interblobs.

A related more general phenomenon is the coincidence of $\mathrm{CO}$ activity with sites of direct thalamic input, as proposed by WongRiley (1979), Livingstone and Hubel (1982), Fitzpatrick et al. (1983), and Martin (1988) for review. The blobs receive afferents from the koniocellular layers of the lateral geniculate nucleus, whereas parvocellular and magnocellular layers project to cortical layers IVc and VI (Fitzpatrick et al., 1983; Hendry and Yoshioka, 1994; Ding and Casagrande, 1997). However, it does not seem to be the quantity of synapses alone, but their quality that relates to an increase in $\mathrm{CO}$ activity, given the fact that thalamic afferents account for only $\sim 5 \%$ of the total amount of excitatory synapses in layer IV in V1 of cats (Ahmed et al., 1994). For layer IVc of V1 of the macaque, a thalamic proportion of $\sim 5-10 \%$ of all synapses was reported by Garey and Powell (1971), and Latawiec et al. (2000) estimate that parvocellular afferents build $\sim 8 \%$ of all asymmetric synapses there. In V2, thick and pale stripes receive afferents from the same source in V1 (Sincich and Horton, 2002) with the pale stripes in fact getting most of these. However, V2 also receives a major projection from the pulvinar, which matches the distribution of CO activity in V2 (Livingstone and Hubel, 1982; Levitt et al., 1995). Furthermore, Land and Erickson (2005) reported that CO-dark subbarrel domains contain high densities of thalamocortical terminals as shown with a marker for VGluT2 in the rat. In several species, a honeycomblike pattern was found at the border between neocortical layers I and II, where CO activity and thalamocortical terminations coincide (Ichinohe et al., 2003).

These findings are in accord with the results of Borowsky and Collins (1989): capillary density paralleled glucose utilization and to some extent $\mathrm{CO}$ activity; furthermore, the latter coincided spatially with synaptic input from the thalamus. Interestingly, vascular density correlated negatively with lactate dehydrogenase activity. Therefore, different metabolic activity patterns (i.e., the 
spatial distribution of oxidative and glycolytic enzymes) might be linked to specific cortical input pathways. The specialized properties of thalamocortical synapses are consistent with this notion. Although the number of thalamic synapses in relation to the excitatory synapses from intracortical sources is small, their impact on the cortical network is significant (Douglas and Martin, 2007). Increased CO activity in thalamic projection sites might arise from the concerted activity of thalamic axon terminals or the postsynaptic properties of their target neurons.

Another noteworthy result of the present study is the different number of perpendicular vessels in blobs and interblobs. Again, there is a discrepancy between previously published data (Zheng et al., 1991) and our own. First, our quantification yielded numbers that are approximately three times higher than in the study by Zheng et al. (1991), and second the local preference of the vertical vessels was the opposite of the previous report (i.e., we found significantly more perpendicular vessels in interblob areas). These discrepancies might arise from the different parameters used for counting. We did not use the diameter of the vessels as a criterion, but their direction with respect to the cortical surface and their morphological characteristics. The vessel caliber was evaluated in a later stage of the analysis. There are different categories of cortical feeding arteries and draining veins defined by their depth of penetration before ramification to capillaries (Duvernoy et al., 1981). The perpendicular orientation of the vessels, clearly visible in tangential sections (Fig. $2 B$ ), and the characteristic space around these vessels void of capillaries (Pfeifer, 1930; Lierse, 1963; Saunders and Bell, 1971; Duvernoy et al., 1981) were taken as basis for the determination of feeding and draining blood vessels, without taking into account their diameter. It has to be noted that this methodology inevitably underestimates vessel numbers because of the fact that the smallest feeding and draining vessels do not even penetrate layer II/III, from which the tangential sections were taken. However, vessels that already tapered to a small caliber when reaching layer II/III were registered with our approach. This might explain the discrepancy between our data and those published by Zheng et al. (1991), where only perpendicular vessels with a diameter $>20$ $\mu \mathrm{m}$ were counted. As shown in Figure $4 E$, the threshold set has a great impact on the outcome of the quantification. One possible conclusion is that perpendicular vessels in general (i.e., regardless of their size) are preferentially located between blobs, whereas large perpendicular vessels tend to be located within blobs.

In summary, blobs undoubtedly show a higher metabolic activity, as seen by $\mathrm{CO}$ histochemistry, and a small but significantly increased vascular density. This probably reflects the higher metabolic capacity related to the anatomical and functional features of the blob. Unfortunately, the difference in vascular density between blobs and interblobs is small, and the noninvasive neuroimaging techniques of today are unable to detect it.

\section{References}

Adams DL, Sincich LC, Horton JC (2007) Complete pattern of ocular dominance columns in human primary visual cortex. J Neurosci 27:10391-10403.

Ahmed B, Anderson JC, Douglas RJ, Martin KA, Nelson JC (1994) Polyneuronal innervation of spiny stellate neurons in cat visual cortex. J Comp Neurol 341:39-49.

Attwell D, Laughlin SB (2001) An energy budget for signaling in the grey matter of the brain. J Cereb Blood Flow Metab 21:1133-1145.

Baborie A, Kuschinsky W (2006) Lack of relationship between cellular density and either capillary density or metabolic rate in different regions of the brain. Neurosci Lett 404:20-22.

Borowsky IW, Collins RC (1989) Metabolic anatomy of brain: a compari- son of regional capillary density, glucose metabolism, and enzyme activities. J Comp Neurol 288:401-413.

Brodmann K (1904-1905) Beiträge zur histologischen Lokalisation der Grosshirnrinde-Dritte Mitteilung: Die Rindenfelder der niederen Affen. J Psychol Neurol 4:177-226.

Carroll EW, Wong-Riley MT (1984) Quantitative light and electron microscopic analysis of cytochrome oxidase-rich zones in the striate cortex of the squirrel monkey. J Comp Neurol 222:1-17.

Ding Y, Casagrande VA (1997) The distribution and morphology of LGN K pathway axons within the layers and CO blobs of owl monkey V1. Vis Neurosci 14:691-704.

Douglas RJ, Martin KA (2007) Mapping the matrix: the ways of neocortex. Neuron 56:226-238.

Duvernoy HM, Delon S, Vannson JL (1981) Cortical blood vessels of the human brain. Brain Res Bull 7:519-579.

Fitzpatrick D, Itoh K, Diamond IT (1983) The laminar organization of the lateral geniculate body and the striate cortex in the squirrel monkey (Saimiri sciureus). J Neurosci 3:673-702.

Fukuda S, Fini CA, Mabuchi T, Koziol JA, Eggleston LL Jr, del Zoppo GJ (2004) Focal cerebral ischemia induces active proteases that degrade microvascular matrix. Stroke 35:998-1004.

Garey LJ, Powell TP (1971) An experimental study of termination of lateral geniculo-cortical pathway in cat and monkey. Proc R Soc Lond B Biol Sci 179:41-63.

Goense JB, Zappe AC, Logothetis NK (2007) High-resolution fMRI of macaque V1. Magn Reson Imaging 25:740-747.

Hamann GF, Okada Y, Fitridge R, del Zoppo GJ (1995) Microvascular basal lamina antigens disappear during cerebral ischemia and reperfusion. Stroke 26:2120-2126.

Hendrickson AE, Hunt SP, Wu JY (1981) Immunocytochemical localization of glutamic acid decarboxylase in monkey striate cortex. Nature 292:605-607.

Hendry SH, Yoshioka T (1994) A neurochemically distinct 3rd channel in the macaque dorsal lateral geniculate nucleus. Science 264:575-577.

Horton JC, Hedley-Whyte ET (1984) Mapping of cytochrome oxidase patches and ocular dominance columns in human visual cortex. Philos Trans R Soc Lond B Biol Sci 304:255-272.

Horton JC, Hubel DH (1981) Regular patchy distribution of cytochrome oxidase staining in primary visual cortex of macaque monkey. Nature 292:762-764.

Humphrey AL, Hendrickson AE (1983) Background and stimulus-induced patterns of high metabolic activity in the visual cortex (area 17) of the squirrel and macaque monkey. J Neurosci 3:345-358.

Ichinohe N, Fujiyama F, Kaneko T, Rockland KS (2003) Honeycomb-like mosaic at the border of layers 1 and 2 in the cerebral cortex. J Neurosci 23:1372-1382.

Jolivet R, Magistretti PJ, Weber B (2009) Deciphering neuron-glia compartmentalization in cortical energy metabolism. Front Neuroenergetics 1:4.

Land PW, Erickson SL (2005) Subbarrel domains in rat somatosensory (S1) cortex. J Comp Neurol 490:414-426.

Landisman CE, Ts'O DY (2002) Color processing in macaque striate cortex: relationships to ocular dominance, cytochrome oxidase, and orientation. J Neurophysiol 87:3126-3137.

Latawiec D, Martin KA, Meskenaite V (2000) Termination of the geniculocortical projection in the striate cortex of macaque monkey: a quantitative immunoelectron microscopic study. J Comp Neurol 419:306-319.

Levitt JB, Yoshioka T, Lund JS (1995) Connections between the pulvinar complex and cytochrome oxidase-defined compartments in visual area V2 of macaque monkey. Exp Brain Res 104:419-430.

Lierse W (1963) Über Die Beeinflussung der Hirnangioarchitektur Durch Die Morphogenese. Acta Anat 53:1-54.

Livingstone M, Hubel D (1988) Segregation of form, color, movement, and depth: anatomy, physiology, and perception. Science 240:740-749.

Livingstone MS, Hubel DH (1982) Thalamic inputs to cytochrome oxidaserich regions in monkey visual cortex. Proc Natl Acad Sci USA 79:6098-6101.

Livingstone MS, Hubel DH (1984) Anatomy and physiology of a color system in the primate visual cortex. J Neurosci 4:309-356.

Lu HD, Roe AW (2008) Functional organization of color domains in V1 and V2 of macaque monkey revealed by optical imaging. Cereb Cortex 18:516-533. 
Martin KA (1988) From enzymes to visual perception: a bridge too far? Trends Neurosci 11:380-387.

Murphy KM, Jones DG, Van Sluyters RC (1995) Cytochrome oxidase blobs in cat primary visual cortex. J Neurosci 15:4196-4208.

O'Kusky J, Colonnier M (1982) A laminar analysis of the number of neurons, glia, and synapses in the adult cortex (area 17) of adult macaque monkeys. J Comp Neurol 210:278-290.

Patel U (1983) Non-random distribution of blood vessels in the posterior region of the rat somatosensory cortex. Brain Res 289:65-70.

Pfeifer RA (1930) Grundlegende Untersuchungen für die Angioarchitektonik des menschlichen Gehirns. Berlin: Verlag von Julius Springer.

Polyak S (1957) The vertebrate visual system. Chicago: University of Chicago.

Russ J, Dehoff R (2000) Practical stereology. New York: Plenum.

Saunders RL, Bell MA (1971) X-ray microscopy and histochemistry of human cerebral blood vessels. J Neurosurg 35:128-140.

Sincich LC, Horton JC (2002) Pale cytochrome oxidase stripes in V2 receive the richest projection from macaque striate cortex. J Comp Neurol 447:18-33.

Sincich LC, Horton JC (2005) The circuitry of V1 and V2: integration of color, form, and motion. Annu Rev Neurosci 28:303-326.

Trusk TC, Kaboord WS, Wong-Riley MT (1990) Effects of monocular enucleation, tetrodotoxin, and lid suture on cytochrome oxidase reactivity in supragranular puffs of adult macaque striate cortex. Vis Neurosci 4:185-204.

Tsai PS, Kaufhold JP, Blinder P, Friedman B, Drew PJ, Karten HJ, Lyden PD, Kleinfeld D (2009) Correlations of neuronal and microvascular densities in murine cortex revealed by direct counting and colocalization of nuclei and vessels. J Neurosci 29:14553-14570.
Ts'O DY, Gilbert CD (1988) The organization of chromatic and spatial interactions in the primate striate cortex. J Neurosci 8:1712-1727.

Weber B, Keller AL, Reichold J, Logothetis NK (2008) The microvascular system of the striate and extrastriate visual cortex of the macaque. Cereb Cortex 18:2318-2330.

Wong-Riley M (1979) Changes in the visual system of monocularly sutured or enucleated cats demonstrable with cytochrome oxidase histochemistry. Brain Res 171:11-28.

Wong-Riley MT (1989) Cytochrome oxidase: an endogenous metabolic marker for neuronal activity. Trends Neurosci 12:94-101.

Wong-Riley MT, Welt C (1980) Histochemical changes in cytochrome oxidase of cortical barrels after vibrissal removal in neonatal and adult mice. Proc Natl Acad Sci U S A 77:2333-2337.

Woolsey TA, Rovainen CM (1991) Whisker barrels: a model for direct observation of changes in the cerebral microcirculation with neuronal activity. In: Brain work and mental activity: quantitative studies with radioactive tracers (Lassen NA, Ingvar DH, Raichle ME, Friberg L, eds), pp 189-198. Copenhagen: Munksgaard.

Yoshioka T, Dow BM (1996) Color, orientation and cytochrome oxidase reactivity in areas $\mathrm{V} 1, \mathrm{~V} 2$ and $\mathrm{V} 4$ of macaque monkey visual cortex. Behav Brain Res 76:71-88.

Zaitsev AV, Gonzalez-Burgos G, Povysheva NV, Kröner S, Lewis DA, Krimer LS (2005) Localization of calcium-binding proteins in physiologically and morphologically characterized interneurons of monkey dorsolateral prefrontal cortex. Cereb Cortex 15:1178-1186.

Zheng D, LaMantia AS, Purves D (1991) Specialized vascularization of the primate visual cortex. J Neurosci 11:2622-2629. 